\title{
DATA TYPES IN COMPUTATIONAL PHONOLOGY
}

\author{
liwan Klein \\ University of Edinburgh, Centre for Cognilive Science \\ 2 Buccleuch Place, Edinburgh EII8 91,W, Scotland \\ Email: kleinoed.ac.uk
}

\begin{abstract}
ABSTRAC'1
This paper examines certain aspects of phonological structure from the viewpoint of abstract data types. Our immediate goal is to find a format for phonological representation which will be reasonably fajthful to the concerns of theoretical phonology while being rigorous enough to admit a computational interpretation. The longer term goal is to incorporate such representations into an appropriate general framework for natu. ral language processing. '
\end{abstract}

\section{Introduction}

One of the dominant paradigms in current conputational linguistics is provided by unificationbased grammar formalisms. Such formalisms (cf. (Shieber 1986; Kasper \& Rounds 1986)) describe hierarchical feature structures, which in many ways would appear to be an ideal setting for formal phonological analyses. Feature bundles have long been used by phonologists, and nore recent work on so-called feature geonetry (c.p. (Clements 1985; Sagey 1986)) has introduced hierarchy into such representations. Nevertheless. there are reasons to step back fron standard feature-based approaches, and instead to adopt the algebraic perspective of abstract data types ( $A D^{\prime}{ }^{\prime}$ ) which has been widely adopted in conputer science. One general motivation. which we shall not explore here, is that the activity of grammar writing. viewed as a process of programme specification. should be anenable to stepwise refinement in which the set of f not neas. sarily isomorphic) models admitted by a loose

\footnotetext{
${ }^{3}$ The work reported in this paper has beoll (ar ried ont as part of the research progranmes of the Human Communication Research Centre supported by the $\mathrm{Ut}$ Economic and Social hesearch (Conncil and the project Computational Phonology: A Constraint-Based Approach, funded by the uk Science and Eingineerimg Research Cound, under grant CiR/6-2084. I an graleful to Steven Bird, Kimba kewton and Tony Simon for discussions relating to this work.
}

specification is gradually narrowed down to a unique algebra (cf. (Sannella \& Tarlecki 1987) for an overview, and (Newton in prep.) for the application to grammar writing). A second motivation, discussed in detail by (Beierle \& Pletat 1988; Beicrle \& Pletal 1989; Beierle et al. 1988), is to use equational ADTs to provide a mathematical foundation for feature structures. A third motivation, dominant in this paper, is to use the Als approach to provide a richer array of explicit. data types than are readily admitted by 'pure' feature structure approaches. Briefly, in their raw form, feature terms (i.e., formalisms for describing feature structures) do not always provide a perspicuous format for representing structure.

On the ADT approach, complex data types axe luilt up from atomic types by means of constructor functions. For example, ... (where we use the underscore ' - to mark the position of the function's arguments) creates elements of type List. A data type may also have selector functions for taking data elements apart. Thus, selectors for the type List are the functions first and last. Standard feature-based encoding of lists uses only selectors for the data type; i.e. the feature labels FIRST and LAST in

\section{(1) FIRST : $\sigma_{1}$ ПLAST : (FIRST $: \sigma_{2} \cap$ LAST : nil)}

However, the list constructor is left implicit. That is, the feature term encoding tells you how lists are pulled apart. but does not say how they are built ujs. When we confine our attention just to lists, this is not much to worry about. However. the situation becones less satisfactory when we attempt to encode a larger variety of data structures into one and the same feature term; say, for example, standard lists, associative lists (i.e. strings), constituent structure hierarchy, and autosegmental association. In order to distinguish adequately between elements of such data types, we really need to know the logical properties of their respective constructors. and this is awk- 
ward when the constructors are not made explicit. For computational phonology, it is not an unlikely scenario to be confronted with such a variety of data structures, since one may well wish to study the complex interaction between, say, non-linear temporal relations and prosodic hierarchy. As a vehicle for computational implementation, the uniformity of standard attribute/value notation is extremely useful. As a vehicle for theory development, it can be extraordinarily unperspicuous.

The approach which we present here treats phonological concepts as abstract data types. $A$ particularly convenient development environment is provided by the language OBJ (Goguen $\&$ Winkler 1988), which is based on order sorted equational logic, and all the examples given below (except where explicitly indicated to the contrary) run in the version of OBJ3 released by $5 \mathrm{RI}$ in 1988. The denotational semantics of an OB.J module is an algebra, while its operational semantics is based on order sorted rewriting. 11.1 and 1.2 give a more detailed introduction into the formal framework, while $\$ 2$ and 3 illustrate the approach with some phonological examples.

\subsection{Abstract Data Types}

A data type consists of one or more domains of data items, of which certain elements aic designated as basic, together with a set of operations on the domains which suffice to generate all data items in the domains from the basic items. A data type is abstract if it is independent of any particular representational scheme. A fundamental claim of the ADJ group (cf. (Goguen. Thatcher \& Wagner 1976)) and much subse(quent work (cf. (Ehrig \& Mahr 1985)) is that abstract data types are (to be modelled as) algebras: and moreover, that the models of abstract data types are initial algebras. ${ }^{2}$

The signature of a many-sorted algebra is a pair $\Sigma=\langle S, O\rangle$ consisting of a set $S$ of sorts and a set $O$ of constant and operation symbols. A specification is a pair $\langle\Sigma \varepsilon\rangle$ consisting of a signature together with a set $\mathcal{E}$ of equations over terms constructed from symbols in $O$ and variables of the sorts in $S$. A model for a specification is

\footnotetext{
${ }^{2}$ An initial algebra is characterized uniquely uj to inomorphism as the semantics of a specificution: there is a unique homamorphism from the intitial algebra inte every algebra of the specification.
}

an algebra over the signature which satisfies all the equations $\mathcal{E}$. Initial algebras play a special role as the semantics of an algebra. An initial algebra is minimal. in the sense expressed by the principles 'no junk' and 'no confusion'. 'No junk" means that the algebra only contains data which are denoted by variable-free terms built up from operation symbols in the signature. 'No confusion' means that two such terms $t$ and $t^{\prime}$ denote the same object in the algebra only if the equation $t=t^{\prime}$ is derivable from the equations of the specification.

Specifications are written in a conventional format consisting of a declaration of sorts, operation symbols (op), and equations (eq). Preceding the equations we list all the variables (var) which figure in them. $\Lambda$ s an illustration, we give below an OB.I specification of the data type LIST1.

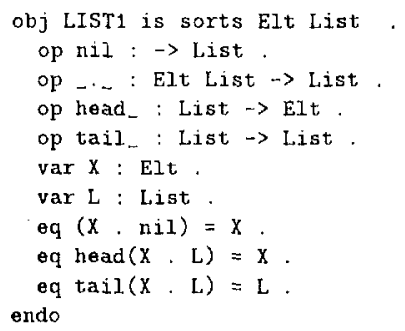

The sort list between the : and the $\rightarrow$ in an operation declaration is called the arity of the operation, while the sort after the $\rightarrow$ is its value sort. Together. the arity and value sort constitute the rank of an operation. The declaration op nil : $\rightarrow$ Elt means that nil is a constant of sort Elt.

Tho specification (2) fails to guarantee that there are any objects of E.1t. While we could of course add some constants of this sort, we would like to have a more general solution. In a particular application. we might want to define phonological words as a List of syllables (plus other constraints of course). ald phonological phrases as a List of words. That is, we need to parameterize the type LIST1 with respect to the class of elements which constitute the lists.

Before turning to parameterization, we will first see how a many-sorted specification language is generalized to an order sorted language by introducing a subsort relation.

Suppose, for example, that we adopt the claim 


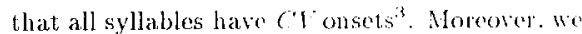
wish to divide syllables into the subclasses leary and light. Obviously we want heary and light syllables to inherit the properties of the class of all syllables, e.g., they have ('l'onsets. We use Heavy < Syll to stale that Heavy is a subsort of the sort Syll. We interpret this to mean that the class of leavy syllables is a subsed of the class of all syllables. Now, let onset_ : Syll $\rightarrow$ Mora be all operation which selects the first mora of a syllable, and let us impose the following constraint (where $\mathrm{CV}$ is a subsort of Mora):

(3) $\operatorname{var} \mathrm{S}: \mathrm{Sy} 1 \mathrm{l}, \operatorname{var} \mathrm{CV}: \mathrm{Cv}$. eq onset $S=C V$

Then the framework of order sorted algetra ensures that onset is also defined for objertin of sent Heavy.

Returning to lists, the specification in (.1) (s)ightty simplified from that used by (Coguen of Winliker 1988 )) introduces Elt and Nelist (nom-empty lists) as subsorts of List. and thereby improves on LIST1 in a number of respects. In addition. the specification is parameterized. That is. it characterizes a list of $X s$, where the parametor $X$ can be instantiated to any modulo which satisfies the condition TRIV; the later is what (Goguen \& Winkler 1988) call a requirement theory" and in this case simply imposes on any input module that it have a sort which can be mappeed to l loe sort E.It.

(4) obj LIST [X : : TRIV] is sorts List NeList subsorts Elt < Nelist < List .

op nil : $\rightarrow$ List.

op _.. : List List $\rightarrow$ List.

op -. - : Nelist List $\rightarrow$ NeList.

op head_ : Nelist $\rightarrow$ Elt.

op tail_ : Nelist $\rightarrow$ List.

var $X$; EIt

$\operatorname{var} L$ : List.

eq $(x, n i l)=x$

eq $\operatorname{head}(X, L)=X$.

$\theta q \operatorname{tajl}(X, L)=L$.

endo

Notice that the list constructor ... now performs the additional function of apyend. allowing two lists to be concatenated. In addition. the so lectors have been made 'safe'. in the sense that they only apply to objects (i.e.. nonemply lists) for which they give sensible results: for what. in LIST1, would have been the meaning of head(ni1)?

\footnotetext{
${ }^{3}$ Here. the term ONSET refers to the inital mota of it syllable in Ilyman's (] 9 xh) version of the moraic theom.
}

\section{Metrical Trees}

As a further illustration, we give below a specification of the data lype BINTREE. This module has two paraneters both of whose requirement theories are TRIV."

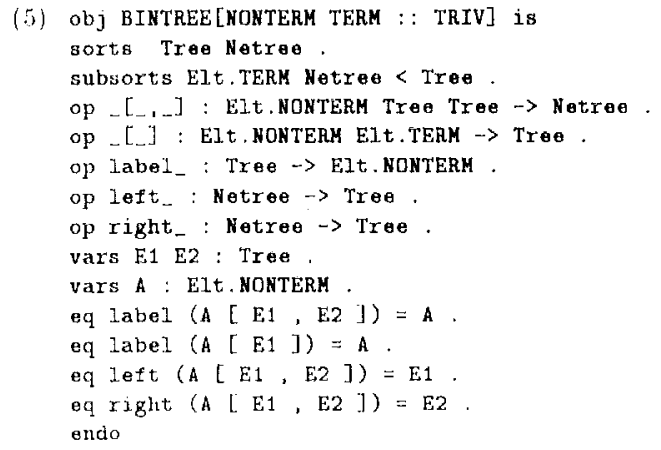

Wo can now instantiate the formal parameters of the module in (5) with input modules which supply appropriate sets of nonterminal and terminal sumbols. Jet us use uppercase quoted identifiers (clements of the (OBI module QID) for nonterminals. and lower case for terninals. The specification in (b) allows us to treat terminals as trees, so that in binary treet. rooted in a node 'A, cant lave lorminals as its langhters. However, we also allow terminals to be directly dominated by a non-branching mother node. Both possibilities occur in the examples belew. (6) illustrates the instantiation of formal parameters by an actual module. namely QID, using the make construct.

(6) make BINTREE-QID is BINTREE[QID,QID] endm

The next example shows some reductions in this module obtailled by treating the equations as rewrite rules applying from left to right.

\footnotetext{
"l'He: ootation Elt . NONTERM. Elt. TERM ntilizes a qual. ification of the sort Elt by the input module's parameter label: this in simply to allow disambiguation.
} 
(7)

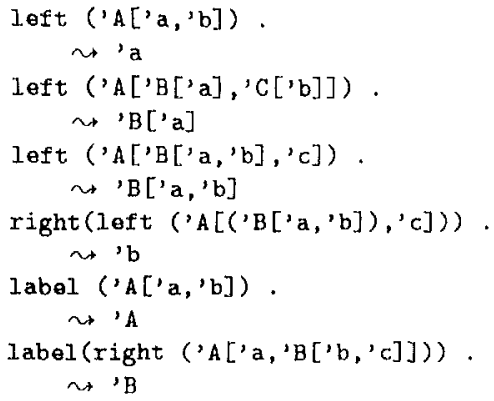

Suppose we now wish to modify the definition of binary trees to obtain metrical trees, These are binary trees whose branches are ordered according to whether they are labelled ' $s$ ' (strong) or 'w' (weak).

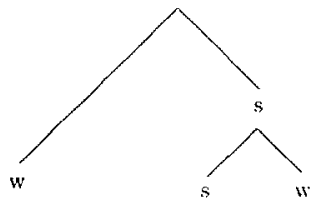

In addition, all trees have a distinguished loaf node called the 'designated terminal element' ( $d t e)$, which is connected to the root of the tres by a path of ' $s$ ' nodes.

Let us define 's' and ' $w$ ' to be our non teminals:

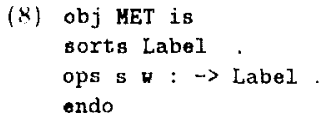

In order to build the data type of metrical trees on top of binary trees, we can import the modulo BINTREE, suitably instantiated, using OB.J's extending construct. Notice that wo Use MET to inntantiate the paraneter which fixes BINTREE's set of non terminal symbols.

(9) obj METTREE is extending BINTREE [MET, QID]* (sort Id to Leaf)

"The * construct tells us that the principal nort of QID. ramely Id, is mapped (by a signalure mopplism) to the sort Leaf in METTREE. ceq signals the presence of a con. ditional equation. $x=$ is a built-in polymorphic equality operation in OBJ.

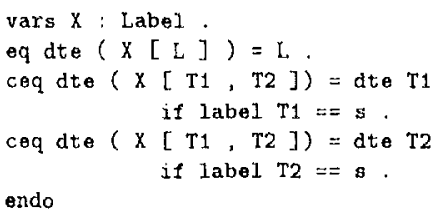

The equations state that the dte (designated terminal element) of a tree is the dte of its strong subtree. Another way of stating this is that the information about dte element of a subtree $T$ is percolated up to its parent node, just in case $T$ is the 's' branch of that node.

The specification METTREE can be criticised on a number of grounds. It has to use conditional equations in a cumbersome way to test which daughter of a binary tree is labelled ' $s$ '. Moreover. it fails to capture the restriction that no binary tree can have daughters which are both weak, or both strong. That is, it fails to capture the essential property of metrical trees, namely that metrical strength is a relational notion.

What we require is a method for encoding the following information at a node: "my left (or right) daughter is strong". One economical method of doing this is to label (all and only) branching nodes in a binary tree witl one of the following two labels: 'sw' (my left daughter is strong), 'ws' (my right danghter is strong). Thus, we replace MET with the following:

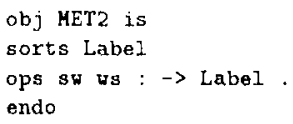

We can now simplify both BINTREE and METREF:

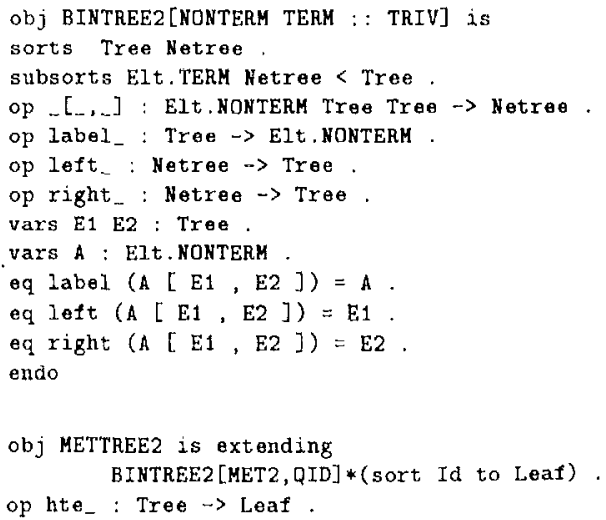


var I. : Leaf ,

vars $T 1$ T2 : Tree.

eq $\operatorname{dte} \mathrm{L}=\mathrm{L}$.

eq dte $T=$ if label $T=s \mathrm{~s}$

then dte (loft $\mathrm{T}$ )

else dte(right $T$ ) $1 i$.

endo

\section{Feature Geometry}

The particular feature geometry we shall specify here is based on the articulatory structure defined in (Browman \& Goldstein 1989). ${ }^{6}$ 'The five active articulators arc grouped into a hierarchical structure involving a tongue node and an oral node, as shown in the followiug diagram.

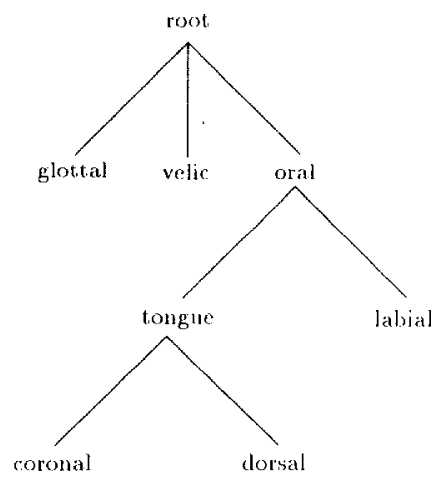

This structure is specilied via term constructors $\{\ldots\}$ and $\{\ldots,-\}$ which give at standard powitional encoding of features. Fach feature valuc is rexpressed as a natural number between 0 and 1 . representing the constriction degree of the corresponding articulator. For example. the term $\{4,0\}$ : Tongue is an item of sort Tongue comsisting of the value th for the feature corosiat. and 0 for the Dorsal; this in turn expresses a situation where there is maximal constriction of the tongue tip, and minimal constriction of the tongue body. Of course, this encoding is rather crude, and possibly sacrifices clarity for concision. However, it suffices as a working exalluple. We will return to constriction degrees belew:

The four sorts Gesture, Root. Oral and Tongue in (10) and the first three operators capture the

\footnotetext{
${ }^{6}$ For space reasons we have omitted any dischenion ob

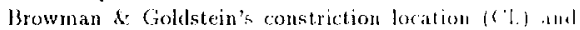
constrietion shape (CS) pirameters. We nlso hase ontitled the supralaryngeal node, since its plomological rob in somewhal dubious.
}

desired tree structure, using an approach which should be familiar by now. For example, the third constructor takes the constriction degrees of Glottal and Velic gestures, and combines them with a complex item of sort Ora1 to build an item of sort Root. The specification imports the module NA'T of natural numbers to provide values for constriction degrees.

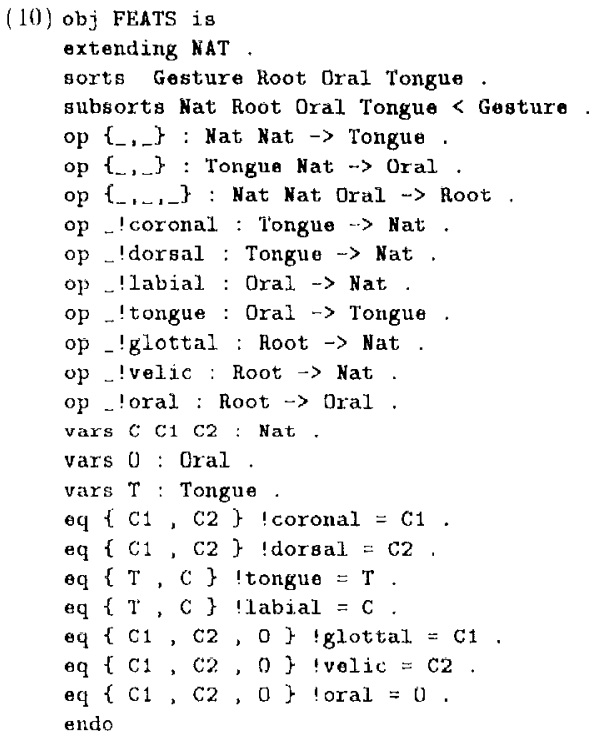

We adopt the notational convention of prepend ing a !" to the name of selectors which correspond directly to features. For example, the coronal selector is a function defined on complex items of sort Tongue which returns an item of sort Nat. representing the constriction degree value for cololality.

Some illustrative reductions in the FEATS module are given below.

$$
\text { (11) } \begin{aligned}
& \{3,4,\{\{4,1\}, 1\}\} \text { !oral. } \\
& \sim\{\{4,1\}, 1\} \\
& \{3,4,\{\{4,1\}, 1\}\} \text { !oral ! torgue. } \\
& \cdots\{4,1\} \\
& \{3,4,\{\{4,1\}, 1\}\} \text { !oral !tongue !coronal. } \\
& \backsim 4
\end{aligned}
$$

In the aro approach to feature structures, reentrancy is represented by equating the values of secectors. Tluts. suppose that two segments S1, S2 slate a coicing specification. We can write thisa a follows: 
(12) S1 !glottal = S2 !glottal

This structure sharing is consistent with one of the main motivating factors behind autosegmental phonology, namely, the undesirability of rules such as $[\alpha$ voice $]-[\alpha$ nasal $]$.

Now we can illustrate the function of selectors in phonological rules. Consider the case of linglish regular plural formation $(-\mathrm{s})$, where the voicing of the suffix segment agrees with that of the immediately preceding segment. unless it is a coronal fricative (in which case there must be an intervening vowel). Suppose we introduce the variables S1 S2 : Root, where S1 is the stenfinal segment and $S 2$ is the suffix. The rule must also be able to access the coronal node of S1. Making use of the selectors. this is simply S2 toral !tongue !coronal (a notation reminiscent of paths in feature logic. (Kasper \& lionnds 1986)). The rule must test whether this coronal node contains a fricative specification. This necessitates an extension to our specification, which is described below.

Browman \& Goldstein (1989. 234ff) define' constriction degree percolation". based on what they call 'tube geometry". The vocal tuact can be viewed as an interconnected set of ubes. and the articulators correspond to valves which have a number of settings ranging from fully open to fully closed. As already mentioned, these settings are called constriction degrees (! cds). where fully closed is the maximal constriction and fully open is the minimal constriction.

The net constriction degree of the oral cavity may be expressed as the maximum of the constriction degrees of the lips, tongue tip and longue body. The net constriction degree of the oral and nasal cavities together is simply the minimum of the two component constriction degrees. To recast this in the present framework is straight forward. However, we need to first define the operations max and min over pairs of natural numbers:

\section{(13) obj MIHHAX}

is protecting NAT.

ops min max : Nat Nat $\rightarrow$ Nat.

vars $M N$ : Nat.

eq $\min (M, N)=$ if $M<=N$ then $M$ else $N$ fi. eq $\max (M, N)=$ if $M>=N$ then $M$ else $N$ fi. endo

(14) obj CD is

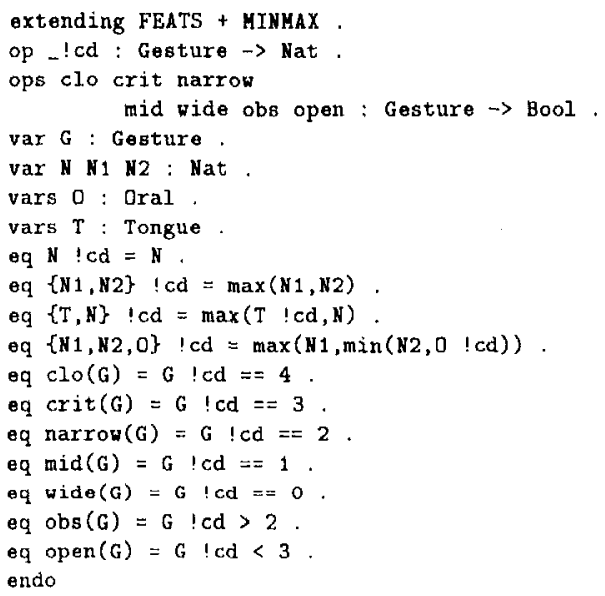

The specification $\mathrm{CD}$ allows classification in to five basic constriction degrees (clo, crit, narrow, mid. and wide) by means of corresponding oneplace predicates. i.e. boolean-valued operations over gestures. For example, the fifth equation above states that $G$ has the constriction degree clo (i.e. $\operatorname{clo}(G)$ is true) if and only if $G ! c d=$ 4.

The working of these predicates is illustrated below:

(15) $\{3,0,\{\{4,1\}, 1\}\}$ !oral !tongue !cd. $\leadsto 4$

$\{3,0,\{\{4,1\}, 1\}\}$ loral !cd. N 4

$\{3,0,\{\{4,1\}, 1\}\}$ !cd.

$\sim 3$

$\operatorname{mid}(\{3,0,\{\{4,1\}, 1\}\}$ !oral !labial). $\sim$ true

wide $(\{3,0,\{\{4,1\}, 1\}\}$ !oral !labial $)$. $\sim$ false

open $(\{3,0,\{\{4,1\}, 1\}\}$ !oral !labial). $\leadsto$ true

$\operatorname{clo}(\{3,0,\{\{4,1\}, 1\}\}$ !oral ! tongue $)$. $\leadsto$ true

\section{References}

Beierle. C. \& I. Pletal (1988). Feature Graphs and Abstract Data Types: A Unifying Approach. Proceedings of the 12 th International Conference on Computational Linguistics. pp40-45, Budapest, Inugary. 
Beierle, C. \& U. Pletat (1988). The Algebra of Feature Graph Specifications. IwHS Roport 94, гнм TR-80.89-029, пвм Germany. Institute for Knowledge Based Systems. Stuttgart.

Beierle, C., U. Pletat \& H. Uszkoreit (1988). An Algebraic Characterization of sTuF.

LILog Report 40, Iвм Germany, Stuttgart.

Bird, S. (1990). Constraint-Based Phonology. PhD Thesis. University of Cdinburgh.

Bird, S. \&. F. Klein (1990). Phonological events. Journal of Linguistics, 26, 33-56.

Browman, C. \& L. Goldstein (1989). Articulatory gestures as phonological units. Phonology, $6,201-251$.

Cardelli, L. (1988) A Semantics of Multiple Inheritance. Information and Computation. $76,138-164$.

Clements, G.N. (1985) The Geometry of Phono logical Features. Phonology Farkot: 2. 225-252.

Dörre, J. \& A. Eisele (1991). A Comprehensive Unification-Based Grammar Lormalism. D) liverable R3.1.B. DYANA - FSPRIT Basic Research Action BR3175, January 1991.

Ehrig, H. \& B. Mahr (1985) Itundementuls of A1gebraic Specification 1: Fiquations and Initial Semantics, Berlin: Springer Verlag.

Goguen, J.A., \& 'T' Winkler (1988) 'ntroducing OBJ3'. Technical Report SHI-(SI-8x-9). SRl International, Computer Science Laboratory, Menlo Park, CA.

Goguen, J.A., J.W. Thatcher and E.G. Wagner (1976) 'An Initial Algebra Approach to the Specification, Correctness and Implementation of Abstract Data Types:. In $R$. Yeh (ed.) Current Trends in Programming Methodology II: Data Structuring. ppso144. Englewood C'liffs, NJ : Prentice IIall.

Hyman, I. M. (1984). On the weightlessness of syllable onsets. In Brugman \& Macanlay (eds.) Proceedings of the Tenth Annual Meeting of the Berkeley Linguistirs Socicty. University of California, Berkeley.
Kasper, R. \& W, Rounds (1986). A rogical Semantics for Feature Structures. Proceedings of the 21h Annual Meeting of the ACL, (olumbia University, New York, NY, 1986, pp 257-265.

Klein, F. (1991). Phonological I)ata Types. In Klein, F, and F'. Veltman (eds) The Dynam. ics of Interpretation: Proceedings of a Symposium on Natural Language and Speech, Brussels, November 26/27, 1991. Springer Verlag.

Newtoll, M. (in preparation). Grammars and Specification Languages. PhD Thesis, Centre for Cognitive Science, University of Edinburgh.

Reape, M. (1991). Foundations of UnificationBased Grammar Formalism. Deliverable ]R.3.2.A. DYANA ESpRT Basic Research Action BR3175, July 1991.

Rounds, W. A A. Manaster-Ramer (1987). A Jogical Version of Functional Grammar. Procctings of 25th Annual Meeting of the Association for Computational Linguistics, 6 9) July 1987. Stanford University, Stanford, $C A, 89-96$.

Sagey, H. (1986). The Representation of Fealures and Rrlations in Non-Linear Phonology. Phl) 'Thesis. MIT', Cambridge, Mass.

Sannella 1). \& $\Lambda$. Tarlecki (L987) Some thoughts on algebraic specilication. lfos Report Serices lics-LFCS-87-21, Laboratory for foundations of Computer Science, University of Jidiuburgh.

Shieber. S. (1986). An Introduction to Unification-Based Approaches to Grammmar. CSII fecture Note Series, University of Chicago Press. Chicago.

Smolka, G, and II. Ait-Kaci (1989) 'Inheritance Ifierarchies: Semantics and Unification', Joumal of Symbolic Computation, 7, $34: 370$. 